\title{
Continuum electrostatic investigations of charge transfer processes in biological molecules using a microstate description
}

\author{
Elisa Bombarda and G. Matthias Ullmann* \\ Received 5th March 2010, Accepted 30th April 2010 \\ DOI: 10.1039/c003905e
}

Charge transfer through biological macromolecules is essential for many biological processes such as for instance photosynthesis and respiration. In these processes, protons or electrons are transferred between titratable residues or redox-active cofactors, respectively. Often their transfer is tightly coupled. Computational methods based on continuum electrostatics are widely used in theoretical biochemistry to analyze the function of even very complex biochemical systems. These methods allow one to consider the $\mathrm{pH}$ and the redox potential of the solution as well as explicitly considering membrane potentials in the calculations. Combining continuum electrostatic calculations with a statistical thermodynamic analysis, it is possible to calculate equilibrium parameters such as protonation or oxidation probabilities. Moreover, it is also possible to simulate reaction kinetics by using parameters calculated from continuum electrostatics. One needs to consider that the transfer rate between two sites depends on the current charge configuration of neighboring sites. We formulate the kinetics of charge transfer systems in a microstate formalism. A unique transfer rate constant can be assigned to the interconversion of microstates. Mutual interactions between sites participating in the transfer reactions are naturally taken into account. This formalism is applied to the kinetics of electron transfer in the tetraheme-subunit and the special pair of the reaction center of Blastochloris viridis. It is shown that continuum electrostatic calculations can be used in combination with an existing rate law to obtain electron transfer rate constants. The relaxation electron transfer kinetics after photo-oxidation of the special pair of photosynthetic reaction center is simulated by a microstate formalism and it is shown to be in good agreement with experimental data. A flux analysis is used to follow the individual electron transfer steps. This method of simulating the complex kinetics of biomolecules based on structural data is a first step on the way from structural biology to systems biology.

\section{Introduction}

Electron transfer in biomolecules often occurs over large distances. Individual electron transfer reactions occur normally only over distances between 5 and $20 \AA$. Electron transfer over larger distances involves several redox centers. Examples for such multi-redox-center systems are complex I, hydrogenase, nitrate reductase, or the photosynthetic reaction center. The charge transfer dynamics in these systems depends strongly on the mutual interaction between the redox active groups. Because electrons are charged particles, this interaction is mainly electrostatic and thus of long range. A similar situation can be found in the case of proton transfer 
reactions which occur also over large distances in hydrogen bonded networks of proteins. Individual proton transfer steps occur normally over distances from 3 to $5 \AA$ A. However, proton transfer can occur over a much larger distance when the transfer takes place in several steps.

The charge transfer dynamics in tightly interacting systems is well described by a microstate model that takes the interaction between the charge transfer sites into account. This model makes it evident that it is not correct to assign single fixed redox potentials or $\mathrm{p} K_{\mathrm{a}}$ values to individual sites of the protein. In fact, because of the charge-charge interaction, the free energy associated with the charge transfer between two sites depends also on the charge state of the surrounding groups. Thus, microscopic free energy differences need to be considered. A consequence of this behavior is that $\mathrm{p} K_{\mathrm{a}}$ values of individual sites in proteins depend on $\mathrm{pH}^{1}$ and redox potentials of individual sites in proteins depend on the solution redox potential.

A well-characterized example of an electron transfer system is the photosynthetic reaction center (RC) from Blastochloris viridis. Its structure has been solved
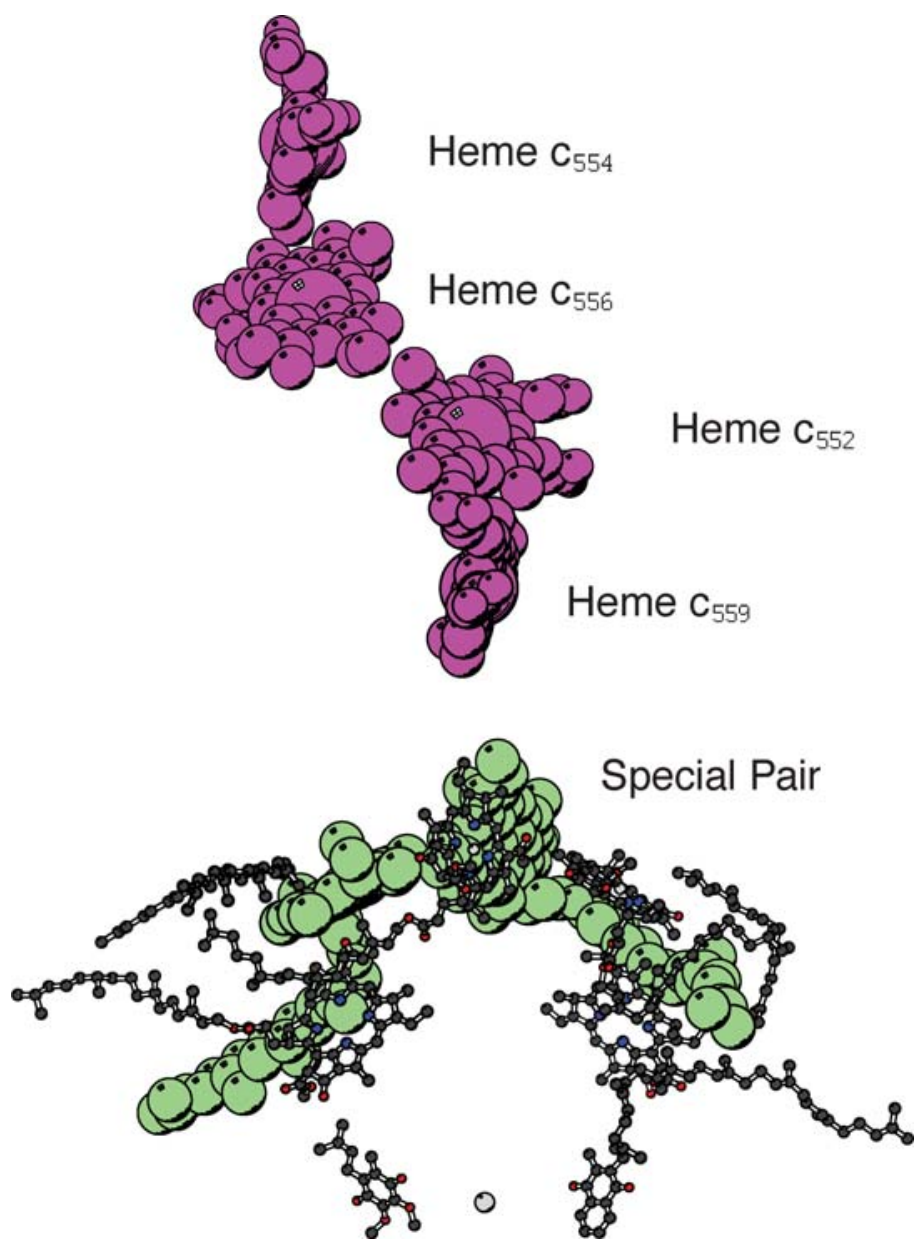

Fig. 1 Cofactors of the RC from B. viridis. The cofactors involved in the electron transfer reaction investigated in this paper (relaxation kinetics after photooxidation of SP) are shown in space-filled mode, the other cofactors as balls-and-sticks. The special pair is shown in green, the hemes are shown in magenta. The hemes are named according to their wavelength of maximal absorbance. 
crystallographically ${ }^{2-4}$ and its equilibrium energetics, as well as its charge transfer kinetics, have been accessed by numerous studies. ${ }^{5-9}$ The $\mathrm{RC}$ from $B$. viridis consists of four subunits labeled $\mathrm{H}, \mathrm{M}, \mathrm{L}$ and $\mathrm{C}$. The three subunits $\mathrm{H}, \mathrm{L}$ and $\mathrm{M}$ facilitate the reduction of a quinone to a quinol. Following the photo-induced excitation of a chlorophyll dimer, the so-called special pair (SP), an electron is transferred via several cofactors to a quinone bound to the so-called $\mathrm{Q}_{\mathrm{B}}$-site. Subunit $\mathrm{C}$ is a tetraheme cytochrome which reduces the oxidized SP (see Fig. 1). Midpoint potentials have been assigned to all four hemes. ${ }^{5,6}$ The heme closest to SP has been identified as the site that primarily re-reduces SP. The kinetics of re-reduction of SP does, however, depend on the charge state of the other heme sites. If prior to the photo-oxidation of SP, the three hemes with the highest midpoint potential are reduced, the re-reduction kinetics of SP is two times faster compared to re-reduction if only one heme is reduced. ${ }^{7}$

In the context of titration experiments, a significant non-Nernst behavior of the titration curve of SP has been reported ${ }^{8}$ This finding was attributed to the electrostatic interaction between SP and heme $c_{559}$. The distinction between mid-point potentials and operational redox potentials was discussed in this context. ${ }^{8}$ Mid-point potentials are redox potentials read as mid-point from titration curves measured in an equilibrium titration experiment, while the operational redox potentials are obtained by inferring the redox potentials from kinetic measurements of electron transfer reactions.

In this article, we present a coherent scheme to simulate electron transport in biological systems. We apply this method to investigate the electron transfer between the SP and the C-subunit after photo-oxidation of the SP of the RC from B. viridis. We call this process relaxation after photo-oxidation. Based on Poisson-Boltzmann electrostatics, electron transfer rates are estimated in the framework of Marcus theory. The rates are calculated for transitions between microstates, i. e., the charge state of the entire system is considered to access the kinetics of electron transfer. This approach allows to use equilibrium electrostatic calculations to access the energetics and mutual interactions between sites and thus provides the kinetically relevant operational redox potentials. The calculations are shown to reproduce well the experimentally determined equilibrium redox potentials of the RC. Furthermore, the changes in re-reduction kinetics of SP upon reduction of additional hemes is reproduced and shown to be due to the electrostatic interaction between heme $c_{559}$ and heme $c_{552}$. In addition, the simulation results obtained with a model including interactions between redox actives sites are compared with a model in which this interaction is neglected. Only the model in which the interactions between the redox sites is explicitly included is able to reproduce all experimental data.

\section{Theory}

It is common to describe charge transfer in proteins as a series of transfer events between the groups involved in the transfer reaction. ${ }^{10,11}$ Accordingly, the electron transfer between the C-subunit and the SP of the RC could be described by estimating rate constants for the transfer between pairs of specific cofactors, like for instance heme $c_{559}$ and SP. This approach, however, bears some problems since the interaction between the sites can not be considered easily. In an alternative approach, the system with many interacting redox-active sites is described by an $N$-dimensional state vector, where $N$ is the number of redox active sites. The elements of this vector are respectively 1 or 0 , indicating whether an electron is bound to the sites (reduced site) or not (oxidized site). Each particular representation of such a vector, for instance $(0,1,1,0,1)$ or $(1,0,1,0,1)$, can be considered as a microstate of the system. The dynamics of the system is described by transition between these microstates. For instance, the transition between $(0,1,1,0,1)$ and $(1,0,1,0,1)$ corresponds to an electron transfer between site 2 and site 1, while site 3 and 5 are reduced and site 4 is oxidized. Accordingly, rates are assigned to transitions 
between microstates and not to transfer events between sites. A description based on microstates is already well established in theoretical calculations of redox and protonation probabilities of proteins at equilibrium ${ }^{12-14}$ and has recently been suggested for the kinetics of charge transfer systems. ${ }^{15-18}$ Here, we use the concept of microstates to describe the kinetics of an electron transfer system. To keep the notation simple, we formulate the theory for a system of redox-active groups only, i. e., an electron transfer system. Treating proton transfer or coupled systems is, however, straightforward within the same theoretical framework.

\section{System description}

We consider a system with $N$ redox-active sites. Each state of the system can be written as an $N$-dimensional vector $\vec{x}=\left(x_{1}, \cdots, x_{N}\right)$, where $x_{i}$ is 0 or 1 if site $i$ is oxidized or reduced, respectively. A transfer event is described as a transition from one state vector to another. In the most general description, transitions are possible between any two given states. In practice, external constraints on the system

a) Photoxidation and Relaxation Processes at Highly Reducing Conditions

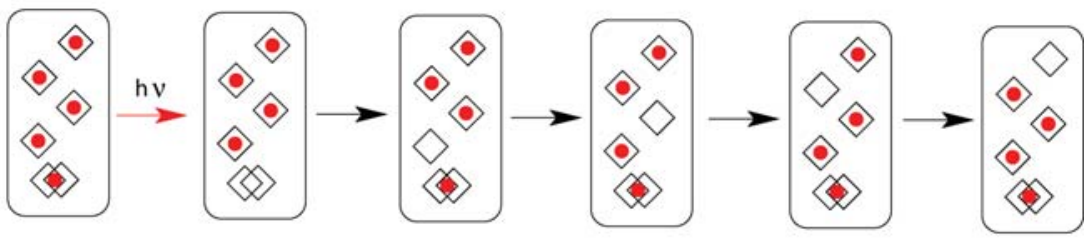

b) Examples for Photoxidation and Relaxation Processes at Less Reducing Conditions

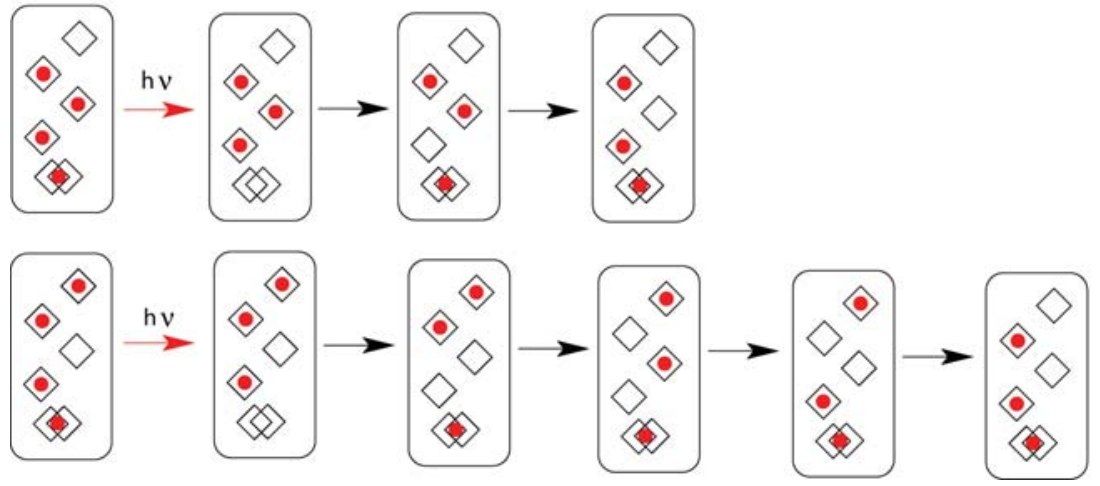

Fig. 2 Examples of electron transfer processes in the photosynthetic reaction center. Each rectangle represents a microstate of the photosynthetic reaction center, the rhombi inside the rectangle represent the hemes and the overlapping rhombi represent the special pair, i.e. it is a schematic representation of Fig. 1. The reduced state of the cofactors is indicated by a red sphere. After photooxidation of the special pair (red arrow), several electron transfer reactions lead to the new equilibrium. The total process is termed relaxation kinetics. Panel (a) shows the processes that occur at highly reducing condition where all hemes are reduced before photooxidation. Panel (b) shows two examples for the relaxation kinetics at less reducing conditions, where one or more hemes are oxidized before photooxidation. These two examples differ in the localization of an oxidized heme. Besides the fact that a different number of steps is required in the two examples to arrive eventually at the same product state, the rates for the transfer reactions between two states will also be different. For instance, the first reaction after photooxidation of the special pair is in both examples an electron transfer from heme $c_{559}$ to the special pair. However, the charge transfer rates are different in the two examples because the nearby heme $c_{552}$ is reduce in the first example and thus has a charge of +2 , while it is oxidized (charge +3$)$ in the second example. The different electrostatic interactions lead to different charge transfer rates. 
may restrict the number of possible transitions. Such restrictions might concern the number of electrons in the system; $i$. e., if no electrons are allowed to enter or leave the system, only transitions between state vectors with equal number of reduced sites will be allowed. We assume that concerted transfer reactions do not occur. Therefore only transitions that represent a single electron transfer are considered. Such transfer events have the form $\left(x_{1}, \cdots, x_{i}, \cdots, x_{j}, \cdots, x_{N}\right) \rightarrow\left(x_{1}, \cdots, \tilde{x}_{i}, \cdots, \tilde{x}_{j}, \cdots, x_{N}\right)$ where $x_{i}$ and $\tilde{x}_{i}$ denote the redox state of site $i$ before and after the transfer event, respectively. These are the processes considered in this paper. Examples for the charge transfer processes are shown in Fig. 2.

Each state of the system has a well-defined energy which depends on the energetics of the individual sites and the interaction between sites. States will be numbered by Greek indices and the roman letters $i$ and $j$ will be used for site indices. The energy of a state $\vec{x}_{\nu}$ is given by: ${ }^{14}$

$$
E\left(\vec{x}_{\nu}\right)=E_{v}=\sum_{i=1}^{N}\left(x_{\nu, i}-x_{i}^{\circ}\right) F\left(\varepsilon_{\mathrm{intr}, \mathrm{i}}^{\circ}-\varepsilon\right)+\frac{1}{2} \sum_{i=1}^{N} \sum_{j=1}^{N}\left(x_{i}-x_{i}^{\circ}\right)\left(x_{j}-x_{j}^{\circ}\right) W_{i j}
$$

where $F$ is the Faraday constant; $x_{\nu, i}$ denotes the redox form of the site $i$ in state $\nu, x_{i}^{\circ}$ is the reference form of site $i ; \varepsilon_{\text {intr, }}^{\circ}$ is the redox potential that site $i$ would have if all other sites are in their reference form (intrinsic redox potential); $\varepsilon$ is the redox potential of the solution; $W_{i j}$ represents the interaction of site $i$ with site $j$.

\section{Equilibrium properties}

Equilibrium properties of a physical system are completely determined by the energies of its states. The equilibrium probability of a single state is given by

$$
p_{\nu}^{e q}=\frac{e^{-\beta E_{v}}}{Z}
$$

with $\beta=1 / k T$ and $Z$ being the partition function of the system.

$$
Z=\sum_{\nu=1}^{M} e^{-\beta E_{\nu}}
$$

The sum runs over all $2^{N}=M$ possible states. Properties of single sites can be obtained from eqn (2) by summing up the individual contributions of all states. For example, the probability of site $i$ being reduced is given by

$$
\left\langle x_{i}\right\rangle=\sum_{\nu}^{M} x_{\nu, i} p_{\nu}^{e q}
$$

where $x_{\nu, i}$ denotes the redox form of site $i$ in the charge state $\nu$.

For a system of interacting sites, the probabilities $\left\langle x_{i}\right\rangle$ can show a complex shape, thus rendering the assignment of mid-point potentials difficult or even meaningless. ${ }^{1,19}$ The energy differences between microstates, however, remain well-defined and thus form a convenient basis to describe the system. It should be noted that the contribution of the solution redox potential cancels for energy differences between state energies (eqn (1)) of two microstates $\mu$ and $\nu$ with the same number of electrons.

\section{Redox equilibrium constants in proteins}

When a protein contains many redox-active groups, one can differentiate between macroscopic and microscopic redox constants (see ref. 1, 19 and 20 for a comparable discussion on protonation constants). The $n$th macroscopic redox potential defines the equilibrium between all states that have $n$ and $(n-1)$ electrons bound. There are in total as many macroscopic redox constants as there are redox-active sites in 
the protein. However, an assignment of a macroscopic redox constant to an individual site is in principle not possible.

Microscopic redox constants describe the equilibrium between two microscopic redox states of the system that differ in the redox-state of one site while the remaining sites do not change their oxidation state. In a protein with $N$ redox-active sites, there are $N 2^{N-1}$ microscopic redox constants of which only $2^{N}-1$ are independent of each other. ${ }^{19}$ For each site, there are $2^{N-1}$ microscopic redox constants which can differ significantly depending on the interaction between the sites. The microscopic redox potential $\varepsilon_{v, i}^{\circ}$ can be calculated from the energy difference of the two respective microstates. Using eqn (1), one obtains

$$
\varepsilon_{\nu, i}^{\circ}=\varepsilon_{\text {intr }, \mathrm{i}}^{\circ}-\frac{1}{F} \sum_{j=1}^{N}\left(x_{\nu, j}-x_{j}^{\circ}\right) W_{i j}
$$

Microscopic $\varepsilon_{v, i}^{\circ}$-values can be assigned to individual sites. However, since for each site there are $2^{N-1}$ microscopic constants, a single microscopic $\varepsilon_{v, i}^{\circ}$-value does not describe the redox-equilibrium of a site appropriately over the entire range of the solution redox potential.

\section{Defining redox constants of individual sites in proteins}

In order to characterize the redox behavior of an individual site of a protein, it is desirable to define a single $\varepsilon^{\circ}$-value that can be uniquely assigned to that site. However, such a definition is not straightforward and deserves some reflection. Two types of such definition seem physically meaningful to us.

Tanford-Roxby $\varepsilon^{\circ}$-value. A possible definition of the redox potential $\varepsilon^{\circ}$ of an individual site can be an average microscopic $\varepsilon^{\circ}$-value. In analogy to the average reduction probability of a site (eqn (4)), an average microscopic $\varepsilon^{\circ}$-value can be defined as

$$
\left\langle\varepsilon_{i}^{\circ}\right\rangle=\sum_{\nu}^{M} \varepsilon_{v, i}^{0} p_{\nu}^{e q}
$$

$p_{v}^{e q}$ is the probability of state $\nu$ as defined in eqn (2). Interestingly, using eqn (5) and (4) it can be shown that eqn (6) is equivalent to eqn (7).

$$
\left\langle\varepsilon_{i}^{\circ}\right\rangle=\varepsilon_{\mathrm{TR}, i}^{\circ}=\varepsilon_{\mathrm{intr}, \mathrm{i}}^{\circ}-\frac{1}{F} \sum_{j=1}^{N}\left(\left\langle x_{j}\right\rangle-x_{j}^{\circ}\right) W_{i j}
$$

This equation is formally similar to an equation proposed by Tanford \& Roxby to calculate the protonation behavior of a protein in an iterative scheme. ${ }^{21}$ In ref. 1 , we have proven that the Tanford-Roxby-p $K_{\mathrm{a}}$ value defines an average microscopic $\mathrm{p} K_{\mathrm{a}}$-value of site $i$. Because of the similarities of $\mathrm{p} K_{\mathrm{a}}$-values and redox potentials of a site ${ }^{14}$ the average microscopic $\varepsilon^{\circ}$-value $\left\langle\varepsilon_{i}^{\circ}\right\rangle$ will be called the Tanford-Roxby (TR) $\varepsilon^{\circ}$-value $\left(\varepsilon_{\mathrm{TR}, i}^{\circ}\right)$. The sum in eqn $(7)$ gives the average energy of interaction with the other sites of the protein, which depends on the average oxidation probability $\left\langle x_{j}\right\rangle$ of the other sites and thus on the solution redox potential. However, it is not considered that also other sites can change their equilibrium oxidation probability when site $i$ changes its oxidation state.

Nernst $\varepsilon^{\circ}$-value. An alternative definition of the $\varepsilon^{\circ}$-value of an individual site can be obtained by rearranging the Nernst equation:

$$
\varepsilon_{\mathrm{N}, i}^{\circ}=\varepsilon+\frac{R T}{F} \ln \frac{\left\langle x_{i}\right\rangle}{1-\left\langle x_{i}\right\rangle}
$$


This redox potential of a site will be called the Nernst $\varepsilon^{\circ}$-value $\left(\varepsilon_{\mathrm{N}, i}^{\circ}\right)$. In analogy to similar definitions of a $\mathrm{p} K_{\mathrm{a}}$-value, ${ }^{1}$ it can be shown that with this definition, the $\varepsilon_{\mathrm{N}, i}^{\circ}$-values relates to the free energy difference of the redox reaction. This definition, besides taking into account the solution redox-potential-dependence of the interactions with other residues, also takes into account that the other sites may also change their average oxidation probabilities.

Meaning of the $\varepsilon^{\circ}$-values of individual sites. Obviously, the two definitions in eqn (6) and (8) are not equivalent. For weak interactions, the difference between the two definitions is almost negligible, but for strong interactions the difference is significant. However, both definitions are meaningful, since they describe different limiting scenarios. The physical picture that is connected to the TR- $\varepsilon^{\circ}$ value (eqn (6)) describes a situation in which the redox-state of all sites is in equilibrium with the solution $\left(\left\langle x_{1}\right\rangle, \ldots,\left\langle x_{i}\right\rangle, \ldots,\left\langle x_{N}\right\rangle\right)_{e q}$. As depicted in eqn (9), the oxidation form of site $i$ is changed from 0 to 1 , while all other sites maintain the average oxidation that they have in equilibrium.

$$
\left(\left\langle x_{1}\right\rangle, \ldots, 0, \ldots,\left\langle x_{N}\right\rangle\right) \rightarrow\left(\left\langle x_{1}\right\rangle, \ldots, 1, \ldots,\left\langle x_{N}\right\rangle\right)
$$

This picture implies that the reduction of site $i$ is faster than the equilibration of the reduction of the surrounding residues.

The physical picture that is connected to the Nernst $\varepsilon^{\circ}$-value (eqn (8)) is related to the free energy difference of oxidation. The reaction starts from a situation in which site $i$ is oxidized and all other sites adapt to this average oxidation probability and ends with site $i$ reduced and again all other sites adapt to the situation (eqn (10)).

$$
\left(\left\langle x_{1}\right\rangle^{\prime}, \ldots, 0, \ldots,\left\langle x_{N}\right\rangle^{\prime}\right)_{e q, \mathrm{ox}} \rightarrow\left(\left\langle x_{1}\right\rangle^{\prime \prime}, \ldots, 1, \ldots,\left\langle x_{N}\right\rangle^{\prime \prime}\right)_{e q, \mathrm{red}}
$$

In this scenario, the reduction of site $i$ is considered to be slow enough to allow all other residues to equilibrate before and after reduction. In contrast to microscopic and macroscopic $\varepsilon^{\circ}$ values which do not depend on the solution redox potential, both definitions of the individual site $\varepsilon^{\circ}$ value lead to solution redox-potentialdependent $\varepsilon^{\circ}$-values. The reason for this dependence is that the interaction between the sites depends on the redox potential of the solution, since the charge of the sites depends on it. Moreover, in the case of the Nernst $\varepsilon^{\circ}$-value, the number of electrons bound to the protein before and after reduction does not necessarily differ by exactly one. From the solution redox-potential-dependence of the two definitions of the individual site $\varepsilon^{\circ}$-values, one can thus conclude that the reduction energy of a site inside a protein with multiple redox sites does not depend linearly on solution redox potential in contrast to the case of a protein with a single redox-active site where the $\varepsilon^{\circ}$-values are independent of solution redox potential. The Nernst $\varepsilon^{\circ}$-value and the TR $\varepsilon^{\circ}$-value represent effective redox potentials of a site; they vary with varying solution redox potential. Often they indicate also the operational redox potentials. In general, however, the operational redox potentials are related to microscopic redox potentials and thus to the microstates of the system.

\section{Time evolution}

The kinetics of the above defined system can be described by a master equation

$$
\frac{\mathrm{d}}{\mathrm{d} t} p_{\nu}(t)=\sum_{\mu=1}^{M} k_{\nu \mu} p_{\mu}(t)-\sum_{\mu=1}^{M} k_{\mu \nu} p_{\nu}(t)
$$

where $p_{\nu}(t)$ denotes the probability that the system is in charge state $\nu$ at time $t, k_{\nu \mu}$ denotes the probability per unit time that the system will change its state from $\mu$ to $\nu$. The summation runs over all possible states $\mu$. For these microstates, energies $E_{\nu}$ and 
transition probabilities $k_{\nu \mu}$ can be assigned unambiguously. The correct time-dependent probability of finding a single site in the reduced form can be obtained by summing up individual contributions from the time-dependent probabilities $p_{\nu}(t)$.

$$
\left\langle x_{i}\right\rangle(t)=\sum_{\nu}^{M} x_{\nu, i} p_{\nu}(t)
$$

Eqn (11) is a system of coupled linear differential equations with constant coefficients. It can be written in the form

$$
\frac{\mathrm{d} \mathbf{p}(t)}{\mathrm{d} t}=\mathbf{A} \mathbf{p}(t)
$$

The diagonal element $A_{\nu \nu}$ of the matrix $\mathbf{A}$ is the negative of the sum over all the rate constants $k_{\mu \nu}$ destroying the state $\nu$. The off-diagonal element $A_{\nu \mu}$ is the rate constant $k_{\nu \mu}$ for the conversion of state $\mu$ to state $\nu .{ }^{16}$ The analytical solution for such equations can be written as

$$
p_{\nu}(t)=\sum_{\mu}^{M} c_{\mu} v_{\mu, \nu} e^{-\alpha_{\mu} t}
$$

where $\alpha_{\mu}$ is the $\mu$ th eigenvalue of the matrix $\mathbf{A}$, and $v_{\mu},{ }_{\nu}$ is the $\nu$ th element of the $\mu$ th eigenvector of matrix $\mathbf{A} ; c_{\mu}$ are integration constants determined from the initial probabilities $\mathbf{p}$ at $t=0$ (i. $e$. all the terms $\left.e^{-\alpha_{\mu} t}=1\right)$.

$$
\mathbf{c}=\mathbf{V}^{-1} \mathbf{p}(0)
$$

where $\mathbf{V}^{-1}$ is the inverse of the matrix containing the eigen vectors of $\mathbf{A}$.

As can be seen from eqn (14), the time dependence of the system is determined by the eigenvalues $\alpha_{\mu}$ of the matrix A. Thus, the initial probabilities of the states do not contribute to the time constants $\alpha_{\mu}$ with which the reaction occur but only to the magnitude $c_{\mu}$ with which these sites contribute to the overall kinetics. In other words, once the eigenvalues and eigenvectors of matrix $\mathbf{A}$ are determined, they are valid for all initial conditions. In the case of an electron transfer system, the initial conditions $\mathbf{p}(0)$ are determined by the solution redox potential. Eqn (15) can then be used to determine the coefficients $\mathbf{c}$ at different redox potentials.

Another interesting feature of this system of equations is that the eigenvalue problem can be solved separately for unconnected reaction subsystems. For instance, if electron uptake is not allowed for the system, the eigenvalue problem can be solved separately for the system with $1,2,3$, or $N$ electrons present. The eigenvalues of these subsystems are independent of each other and the total kinetics is just a linear combination of the kinetics of these subsystems.

To analyze a complex charge transfer system, it is of particular interest to follow the flow of charges through the system, i. e., the charge flux. The flux from state $\nu$ to state $\mu$ is given by the probability of state $\nu$ times the probability per unit time that state $\nu$ will change into state $\mu, i$. e., by $k_{\mu \nu} p_{\nu}(t)$. The net flux $J_{\mu \rightarrow \nu}$ between states $\mu$ and $\nu$ is thus given as the sum of the flux of the forward and the backward reaction.

$$
J_{\mu \rightarrow \nu}(t)=k_{\nu \mu} p_{\mu}(t)-k_{\mu \nu} p_{\nu}(t)
$$

\section{Calculation of the rate constants $\boldsymbol{k}_{\nu \mu}$}

The outlined theory is directly applicable to a large class of reaction systems such as for example proton and electron transfer in proteins. The method to determine the rate constants $k_{\nu \mu}$ will, however, be specific for the particular reactions that should 
be simulated. For electron transfer systems, continuum electrostatic calculations in combination with existing rate laws ${ }^{22,23}$ can be used to obtain electron transfer rates in good agreement with experimental data. Three factors mainly govern the rate constants of biological electron transfer reactions: the energy difference between the donor and acceptor state, the environmental polarization (reorganization energy), and the electronic coupling between the redox sites. The energy barrier for the transfer process is given in the framework of Marcus theory as

$$
\Delta G^{\neq}=\frac{\left(\Delta G^{\circ}-\lambda\right)^{2}}{4 \lambda}
$$

where $\Delta G^{\circ}$ is the energy difference between donor and acceptor state and $\lambda$ is the socalled reorganization energy. The electronic coupling between the redox sites can be accounted for by a distance-dependent exponential function $A \exp \left(-\alpha\left(R-R^{\circ}\right)\right)$ where $R$ is the edge-to-edge distance between cofactors; $R^{\circ}$ represents a van-derWaals contact distance and $A$ represents an optimal rate. These aspects of biological electron transfer have been successfully combined to formulate a heuristic rate law applicable to long range electron transfer: $:^{22,23}$

$$
\begin{gathered}
\log \left(k_{e x}\right)=13-0.6(R-3.6)-3.1 \frac{\left(\Delta G^{\circ}+\lambda\right)^{2}}{\lambda} \\
\log \left(k_{e n}\right)=13-0.6(R-3.6)-3.1 \frac{\left(-\Delta G^{\circ}+\lambda\right)^{2}}{\lambda}-0.06 \Delta G^{\circ}
\end{gathered}
$$

where $k_{e x}$ and $k_{e n}$ are the rate constants for exergonic and endergonic electron transfer reactions, respectively. In these equations suggested by Dutton and coworkers, the reaction energy and the reorganization energy need to be given in $\mathrm{eV}$ and the distance in $\AA$ in order to fit with the numerical coefficients. Note that eqn (19) is a consequence of the relation

$$
k_{e n}=k_{e x} e^{-\beta G^{\circ}}=k_{e x} K_{e q}
$$

where $K_{e q}$ is the equilibrium constant for the equilibrium between the the donor and the acceptor state. The free energy $\Delta G^{\circ}$ for a transition between two states $\mu$ and $\nu$ can be calculated within the electrostatic model using eqn (1), i. e., $E_{\nu}-E_{\mu}$. The reorganization energy $\lambda$ contains two contributions, $\lambda=\lambda_{o}+\lambda_{i}$, where $\lambda_{o}$ is the solvent reorganization energy and $\lambda_{i}$ accounts for changes of the nuclear degrees of freedom between the donor and acceptor sites. $\lambda_{o}$ was shown to be accessible to equilibrium calculations: ${ }^{24,25}$

$$
\lambda_{o}=\frac{1}{2} \sum_{i}^{K}\left(\phi_{a d}^{\mathrm{opt}}\left(\vec{r}_{i}\right)-\phi_{a d}\left(\vec{r}_{i}\right)\right) \Delta q_{i}^{a d}
$$

$\Delta q_{i}^{a d}$ is the change in charge of atom $i$ when going from the donor to the acceptor state. The potentials $\phi_{a d}{ }^{\mathrm{pt}}$ and $\phi_{a d}$ are generated by the charge distribution $\Delta \rho=\rho_{a}-\rho_{d}$ in a low (opt) and in a high dielectric environment, respectively. Here, $\rho_{a}$ and $\rho_{d}$ denote the charge distribution of the acceptor and donor state, respectively. The permittivity constant for the low dielectric environment reflects the electronic polarizability while the permittivity constant for the high dielectric environment accounts for the nuclear and electronic polarizability. The solvent reorganization energy is given by the difference in solvation free energy of the charge distribution $\Delta \rho$ between a low and a high dielectric environment. $\lambda_{i}$ can be estimated by DFT calculations but are often found to be significantly smaller than the solvent reorganization energy. ${ }^{26-29}$ The inner sphere reorganization energy can be calculated from density functional theory as 


$$
\lambda_{i}=E_{\mathrm{bond}}\left(\vec{r}_{d}, \rho_{a}\right)-E_{\mathrm{bond}}\left(\vec{r}_{a}, \rho_{a}\right)
$$

where $E_{\mathrm{bond}}$ is the total DFT energy of the two sites; $\vec{r}_{d}$ and $\vec{r}_{a}$ correspond to the optimized geometry of the sites in the donor and acceptor state, respectively. Hence, the inner sphere reorganization energy is given by the difference in bonding energy between donor and acceptor geometry while the sites are kept in their acceptor state charge distribution. ${ }^{28,29}$

In this formulation, the total reorganization energy depends only on the difference of the charge distribution between two states. In a single electron transfer reaction, only the charge of the donor site and the charge of the acceptor site changes, while the charges of the other sites remain unchanged. Thus, the electron transfer between sites $i$ and $j$ is always connected to the same reorganization energy irrespective of the states that are converted into each other.

\section{Methods}

\section{Structures and parameters}

In the calculations, we used the structures of the $\mathrm{RC}$ from Blastochloris viridis (PDB code $1 \mathrm{PRC})^{30}$ having a resolution of $2.3 \AA$. All water molecules were deleted. Hydrogens were added with HBUILD $^{31}$ in $\mathrm{CHARMM}^{32}$ and subsequently minimized using the CHARMM force field. ${ }^{33}$ For the measurement of the edge-to-edge distance between the cofactors, we considered only the atoms that are part of the aromatic system of the cofactors.

The atomic partial charges for most atoms were taken from the CHARMM force field. ${ }^{33}$ The partial charges of the hemes, the special pair, and the linked residues were obtained from a density functional calculation (functionals $\mathrm{VWN}^{34}$ and PW91 ${ }^{35}$ ) using the ADF program package. ${ }^{36}$ The electrostatic potentials obtained from the density functional calculation were fitted using the CHELPG-algorithm ${ }^{37}$ combined with a singular value decomposition. ${ }^{38}$ The charges of all other cofactors are the same as in previous calculations. ${ }^{39-41}$

\section{Continuum electrostatic calculations and equilibrium redox titration}

All the electrostatic calculations were done using the program suite MEAD ${ }^{12,42}$ which uses a finite difference method to determine the electrostatic potential. For the calculations of the intrinsic redox potentials and the interaction energies, the dielectric constant of the protein and the dielectric constant of water were set to 4.0 and 80.0 , respectively. The ionic strength was set $0.1 \mathrm{M}$. For the protein, the electrostatic potential was calculated by focusing using three grids of $141^{3}, 121^{3}$, and $121^{3}$ grid points and grid spacings of $2.0 \AA, 1.0 \AA$ and $0.25 \AA$, respectively. For the model compounds, the electrostatic potential was calculated by focusing using two grids of $121^{3}$ grid points and grid spacings of $1.0 \AA$ and $0.25 \AA$. The first grid was centered on the protein or the model compound, the other grids were centered on the titratable group.

For the calculation of the reorganization energy, we used a dielectric constant of 1.0 for the cofactor, 2.0 for the electronic dielectric constant, 4.0 for the total dielectric constant of the protein, and 80.0 for the dielectric constant of water. In the calculation of the total reaction field potential, an ionic strength of $0.1 \mathrm{M}$ was considered. The electrostatic potential was calculated by focusing using three grids of $181^{3}, 181^{3}$, and $301^{3}$ grid points and grid spacings of $2.0 \AA, 1.0 \AA$ and $0.25 \AA$, respectively. The first grid was centered on the geometric center of the protein, the other two grids were centered on the geometric center of the cofactors between which the electron transfer takes place.

In order to calculate the energy of the different redox states, we first determined the protonation of the different protonatable residues in the protein using continuum electrostatic and Monte Carlo simulations. ${ }^{13}$ We then fixed the highest 
populated protonation state in order to calculate the energy of the different redox states. The redox potentials of the special pair, of the bis-histidinyl heme and the metioninyl-histidinyl heme were adjusted in order to fit the equilibrium redox behavior. The equilibrium titration curves and the probability of the different redox states in dependence on the solution redox potential have been calculated by a statistical mechanics averaging.

\section{Solution of the differential equation}

In order to solve the system of differential equations in eqn (11) in the form of eqn (12), the eigenvalues and the eigenvectors of the matrix in eqn (11) need to be determined. We used the routine DGEEV of the LAPACK library for this purpose. The coefficients $c_{\mu}$ in eqn (12) are determined from the initial conditions at $t=0$. The linear system of equations of eqn (12) at $t=0$ is solved using standard numerical methods (lower and upper triangular decomposition). ${ }^{43}$

\section{Results and discussions}

In previous work, ${ }^{16}$ we simulated the relaxation kinetics after photooxidation of the special pair in the photosynthetic reaction center using a microstate description. This relaxation kinetics is the transfer of electrons from the tetraheme C-subunit to SP which leads to the re-reduction of SP. In the present work, we complement that approach with the discussion of the energetics of the electron transfer process. In this section, we first discuss equilibrium properties underlining the necessity to take into account the interaction between the sites. Then, after recalling the main results of our previous study ${ }^{16}$ on the time evolution of the electron transfer, we discuss the energy landscapes associated with the electron transfer process. To show the role of the electrostatic interactions between the sites, we compare the simulation of the electron transfer kinetics once considering and once neglecting the interaction between the sites. Finally, we discuss the influence of the solution redox potential on the relaxation kinetics.

\section{Relevant redox potentials of the sites at different solution redox potentials}

The redox-titration curves of all considered redox active groups of the RC seem to have standard sigmoidal shape as described by the Nernst equation of an isolated redox-active group (Fig. 3a). Thus, just from these curves, it seems possible to assign unambiguously a redox potential to each redox active group. However, due to the interaction between the redox active groups, 16 different microscopic redox potentials are associated with each site. A microscopic redox potential is defined as the redox equilibrium between two microstates which differ in the oxidation state of just one site. Since only strong interactions lead to a significant shift of the redox potential and not all sites interact strongly, some of the microscopic redox potentials are very similar (see Fig. 3b-f) which reduces the number of distinguishable microscopic redox potentials.

As discussed in the Theory section, the Nernst and the Tanford-Roxby redox potentials (blue and red curves respectively in Fig. 3b-f) represent an effective site redox potential of a redox-active group at a given solution redox potential. One can see that these two types of effective site redox potentials vary between the most extreme microscopic redox potentials associated with that site. Larger changes in the effective redox potential of a site occur when sites strongly interacting with the site of interest change their oxidation state. For instance, heme $c_{559}$ (Fig. 3c) has a redox potential of about $285 \mathrm{mV}$ at solution redox potentials of about $-200 \mathrm{mV}$. At a solution redox potential of about $0 \mathrm{mV}$, i.e. when heme $c_{552}$ changes its oxidation state (green curve in Fig. 3a), the redox potential changes to $\sim 350 \mathrm{mV}$. At higher solution redox potentials redox $(\sim 450 \mathrm{mV})$, the redox potential of heme $c_{559}$ shift again to a higher value of about $400 \mathrm{mV}$. This change is due to the 


\section{a) Titration curves}

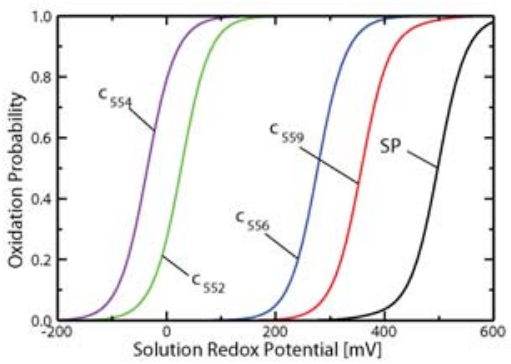

\section{c) Heme $\mathrm{C}_{559}$}

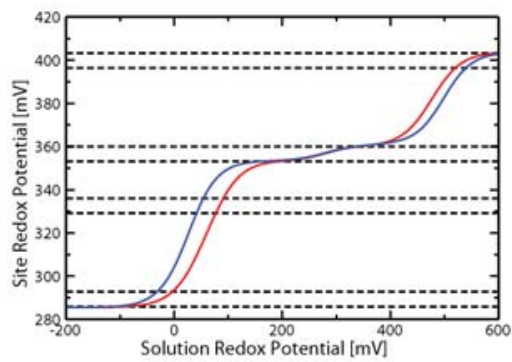

e) Heme $c_{556}$

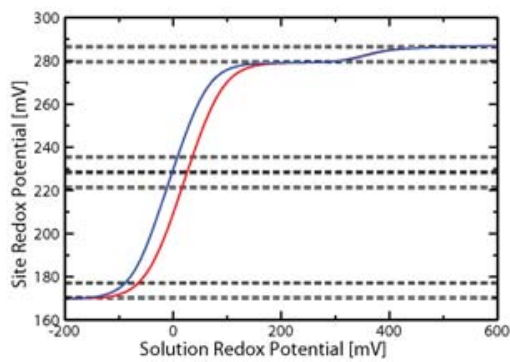

\section{b) Special Pair}

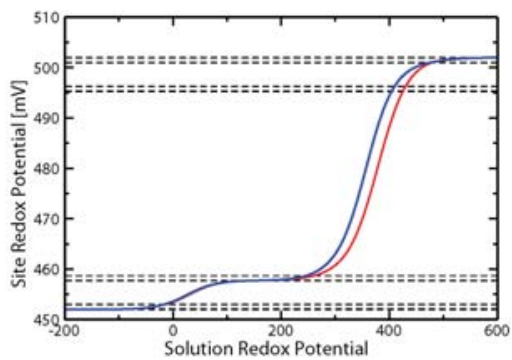

\section{d) Heme $\mathrm{C}_{552}$}

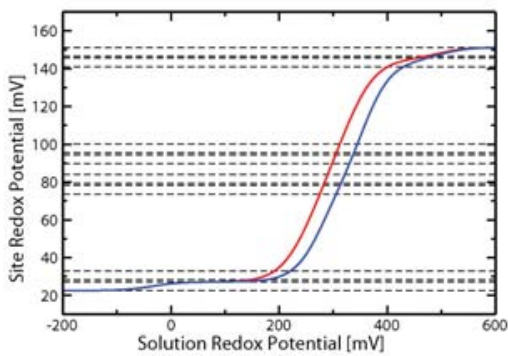

\section{f) Heme $\mathrm{C}_{554}$}

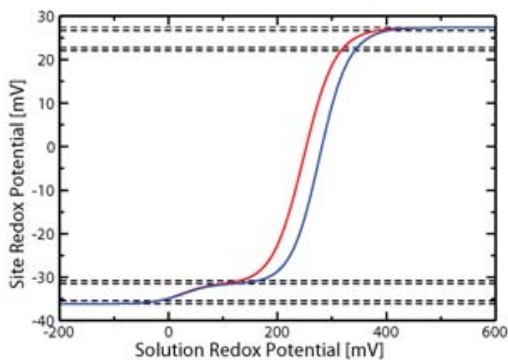

Fig. 3 Redox titration behavior of SP and the hemes of the RC from B. viridis. (a) Probability of the oxidized form of the redox-active sites; (b)-(f) solution redox-potential dependence of the site redox potential of the special pair (b), heme $c_{559}(\mathrm{c})$, heme $c_{552}(\mathrm{~d})$, heme $c_{556}(\mathrm{e})$, heme $c_{554}$ (f). The dashed lines show the different microscopic redox potentials, the blue line and the red line show the Nernst redox-potential and the Tanford-Roxby redox-potential, respectively.

oxidation of SP. Similar observations can be made also for the other sites. This dependence of the redox potential of one site on the charge state of the other redox-active sites and thus on the redox potential of the solution causes that the electron transfer rate between two sites may be different at different solution redox potentials. Moreover, this dependence indicates how the mutual interaction influences the redox properties of the proteins.

\section{Importance of the electrostatic interactions for the description of the electron transfer kinetics}

In order to investigate the importance of the electrostatic interaction for the relaxation kinetics after photo-oxidation, we compare the simulation of the kinetics of two models. In one model, we consider the interaction between the sites and, thus, the 


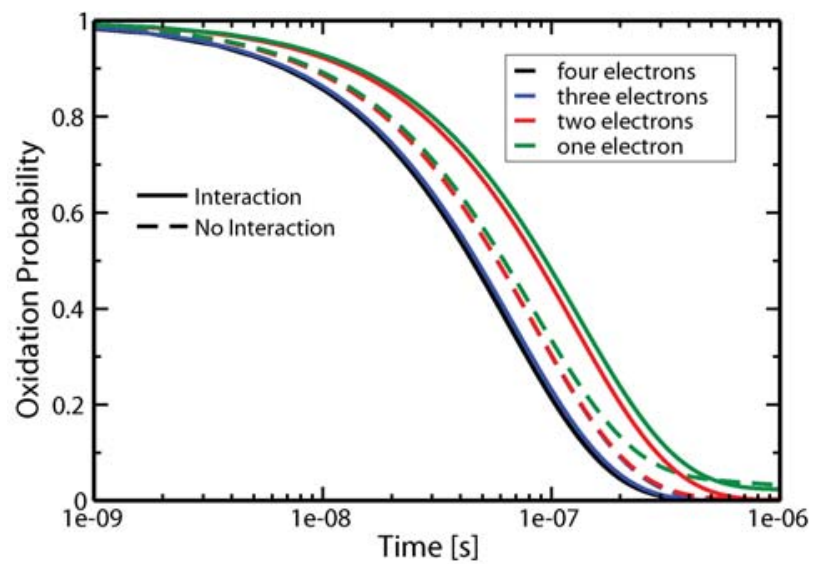

Fig. 4 Re-reduction kinetics of SP. The solid curves show the kinetics of the model in which the interaction between the sites is included, the dashed curves show the kinetics of the model in which this interaction is neglected. Only the simulation in which the interaction is taken into account is able to reproduce the experimentally observed behavior properly, namely that the re-reduction kinetics is faster by a factor of about two when three or four electrons are distributed over the hemes compared to when only one or two electrons are distributed.

electron transfer energetics is calculated using eqn (1). In the other model, we assume that it is possible to read the redox potentials from the titration curves in Fig. 3a; thus we implicitly neglect the interaction between the sites. The reorganization energies were calculated using the Poisson-Boltzmann equation ${ }^{16}$ and they were assumed to be the same in both models.

In Fig. 4, we compare explicitly the re-reduction kinetics of SP for the model with interaction and the model without interaction. Here the differences between the two models are particularly interesting because a direct comparison to experimental numbers is possible. It was shown experimentally that the kinetics with one and two electrons in the system differs very much from the kinetics with three electrons in the system. ${ }^{7}$ As can be seen in Fig. 4, this behavior can be well reproduced when the interaction between the sites is considered (continuous lines). In contrast, the simulations in which the interaction between the sites is neglected (dashed lines), the kinetics is more or less the same irrespective of the number of electrons in the system.

Fig. 5 shows the relaxation kinetics after photo-oxidation of SP for the two models, i.e. in one case (left) with interactions and in the other case (right) without interactions. Despite the general similarity of kinetics, there are obvious differences between the two models. One sees that in the model without interaction (right column in Fig. 5), the transiently populated intermediates have a higher probability. This stronger population of the intermediates is caused by higher barriers in the model without interactions, as will be shown later. This higher barrier led to slower re-reduction kinetics making the model without interaction in disagreement with experiments. For example in Fig. 5a, the model in which the interaction is taken into account is equilibrated after about $0.5 \mu \mathrm{s}$, while it takes about $8 \mu$ s to reach equilibrium after photo-oxidation in the model in which the interaction is neglected. The interactions lead to lower barriers and thus faster relaxation kinetics after photoreduction of the special pair.

In the system with four electrons (Fig. 5a), it seems that the electron transfer occurs from heme $c_{552}$ to the SP and from heme $c_{554}$ to heme $c_{552}$ : when the oxidation level of SP drops, the oxidation level of heme $c_{552}$ rises and when the oxidation level of heme $c_{552}$ drops, the oxidation level of heme $c_{554}$ rises. It seems as though heme $c_{559}$ and heme $c_{556}$ are not involved in this first relaxation kinetics. However, the flux analysis 
with interaction

a) System with four electrons

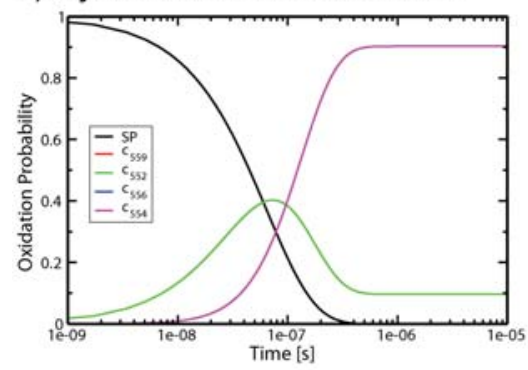

b) System with three electrons

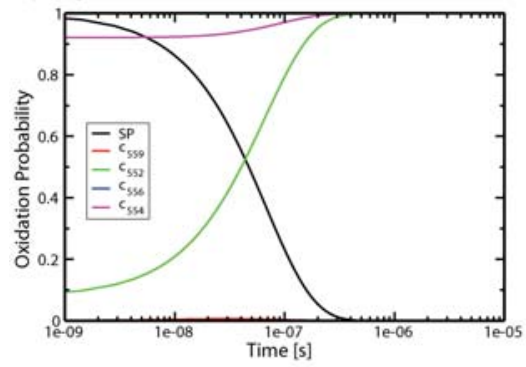

c) System with two electrons

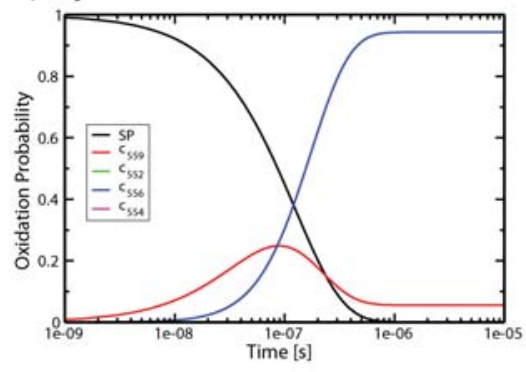

d) System with one electron

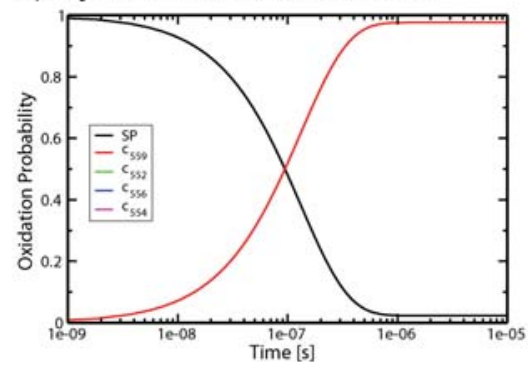

without interaction
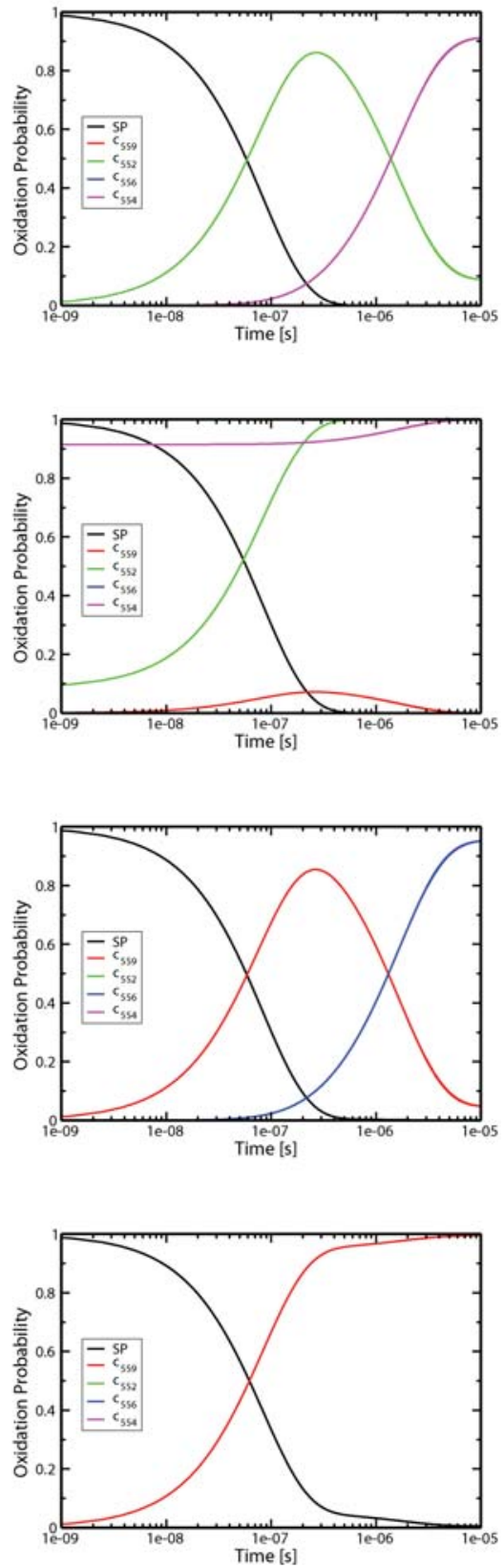

Fig. 5 Time-dependent oxidation probability of the redox active sites of the RC after photooxidation of SP. The left column shows the kinetics of a model in which the interaction between the sites is taken into account. The right column shows the kinetics of a model in which this interaction is neglected. The initial setup consists of 4 (a), 3 (b), 2 (c), or 1 (d) electrons distributed over the four hemes. Initial distributions of the microstates were taken from an equilibrium distribution prior to photo-oxidation of the SP. 
a) System with four electrons

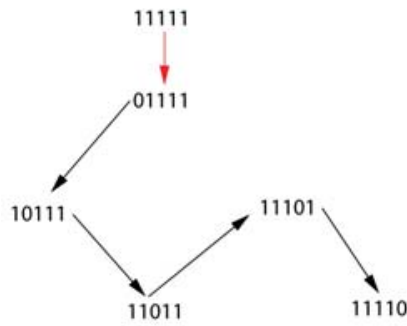

c) Systems with two electrons

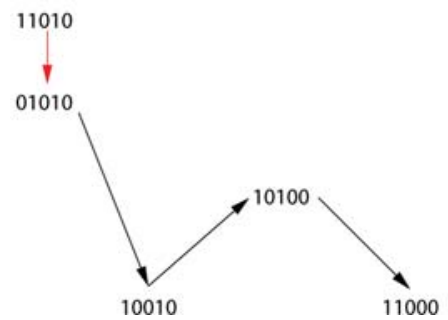

b) System with three electrons

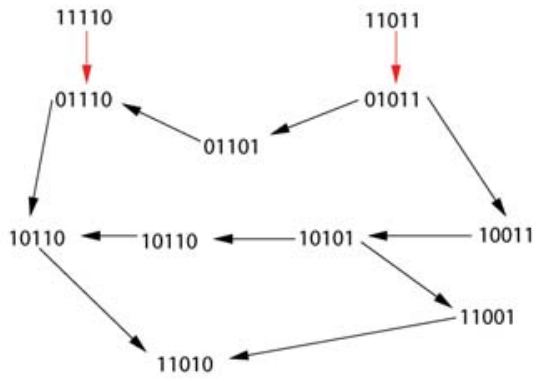

d) System with one electron

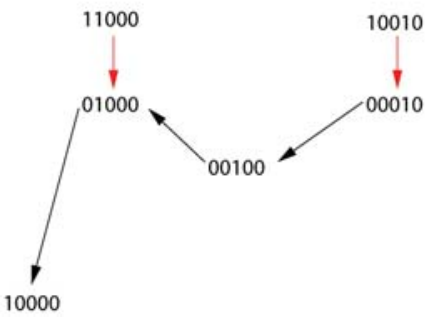

Fig. 6 Scheme of the flux through the various states. The red arrows indicate the photo-oxidation, the black arrows show the electron transfer reactions in the subsequent relaxation process. The schemes are shown for systems with 4 (a), 3 (b), 2 (c), or 1 (d) electrons. The final equilibrium states are shown on the bottom of each scheme. For the system with four and two electrons, two microstates are in equilibrium at the end. Consequently, the relaxation process of the systems with three and one electrons starts from two states.

reveals that heme $c_{559}$ and heme $c_{556}$ are involved. ${ }^{16}$ Due to their redox properties, the oxidation probability of heme $c_{559}$ and heme $c_{556}$ is, however, much less then $1 \%$ even during the relaxation process and thus not visible in the plots in Fig. 5a. In fact, the transfer from heme $c_{554}$ to SP proceeds through heme $c_{559}$ and heme $c_{556}$ since a direct electron transfer from heme $c_{552}$ to SP or from heme $c_{554}$ to heme $c_{552}$ would be much too slow because the large distance between the redox centers.

Similar observations as described for Fig. 5a, can be made for Fig. 5b-d in which the relaxation kinetics for systems with three, two, and one electron are described. In all cases when the interaction is neglected, the relaxation kinetics is slower and intermediates, if existing, have a higher probability of occurrence.

From the kinetic traces in Fig. 5, it is not obvious through which states the reaction occurs. However, the flux analysis using eqn (16) allows us to analyze the detailed pathway of the mechanism. ${ }^{16}$ Fig. 6 shows the mechanism of the relaxation after photo-oxidation of SP as inferred from a flux analysis. The state vectors have the following order: $\mathrm{SP}$, heme $c_{559}$, heme $c_{552}$, heme $c_{556}$, heme $c_{554}$. The relaxation kinetics starts from a single microstate for the system with four and two electrons and ends with two microstates in equilibrium. Consequently, in the systems with three and one electrons, the kinetics starts from two different microstates after photo-oxidation. In the later case, one of the two microstates has a higher probability (for the system with three electrons, the major species is (01110) and for the system with one electron, the major species is (01000)). In the case of the system with three electrons (Fig. 6b), the graph showing the relaxation process displays some complexity, mainly originating from the minor species (01011).

However, many of the microstates that are important for the mechanism of the charge transfer have a very low probability of occurrence during the relaxation process. For instance, for the system with four electrons, the states 10111 and 
11101 have a probability that is much smaller than $1 \%$ during the whole relaxation process, even if the two states are nearly unpopulated for different reasons. For a better understanding, we analyze the energy landscape of the relaxation process (see Fig. 7). Fig. 7a shows the energy landscape of the relaxation of a system with four electrons. One sees that the reaction sequence $01111 \rightarrow 10111 \rightarrow 11011$ is a steep downhill process. The state 10111 does not get highly populated, because it is an intermediate in a steep downhill process. Instead the state 11101 does not get highly populated, because the reaction $11011 \rightarrow 11101$ is an uphill reaction, that is followed by a downhill reaction $11101 \rightarrow 11110$. Thus, this example shows that there might be different reasons why intermediate states are not seen in experimental studies.

An interesting feature when comparing the energy landscapes for the model in which the interaction between the redox sites is considered and the model in which this interaction is neglected, is that the activation energies are smaller, the uphill processes are less uphill and the downhill processes are less downhill in the model in which the interaction is taken into account. Thus the energy landscape in which the interaction between the sites is considered allows a faster electron transfer in which less energy is dissipated. The energy landscape of the system with two electrons (Fig. 7c) is particularly interesting in this respect. In the model considering the interaction, the process $01010 \rightarrow 10010 \rightarrow 10100$ is an overall downhill process, while it is instead an overall uphill process in the model without interactions making this reaction step unfavorable. This difference between the two models emphasizes the importance to consider the interaction between the redox-active sites.

\section{Relaxation kinetics at different solution redox potentials}

In experimental studies, the initial reaction conditions of the relaxation kinetics after photo-oxidation of SP are adjusted by setting the redox potential of the solution, i.e. the protein solution adopts a Boltzmann distribution for the redox states before photooxidation. Then SP gets excited and eventually photooxidized. After photooxidation, the system is in a non-equilibrium situation which then relaxes. Fig. 8a shows the probability of the different redox states before photo-oxidation. The probability of the macroscopic states are shown by solid lines, the probability of the microscopic states by dashed lines. For some of the macrostates, only one microstates is significantly populated and thus the solid line and the dashed line coincide. This is the case for instance in the system with three electrons before photooxidation (blue curve in Fig. 8a). Fig. 8b-f show the relaxation kinetics of SP and the four hemes. As discussed in the Theory section, the overall kinetics of the relaxation can be separated into contributions of systems with four, three, two, and one electron. The overall kinetics shown in Fig. 8 is thus a linear superposition of the kinetics shown in Fig. 5. The contribution of each initial state to the kinetics is determined by the Boltzmann distribution before photo-oxidation.

In Fig. 8a, it can be seen that at a solution redox potential of $-200 \mathrm{mV}$, only one microscopic state, (1111), is populated before the photo-oxidation. Thus the initial state of the relaxation kinetics after photo-oxidation is (01111). The reaction kinetics at this solution redox potential can thus be uniquely assigned to the relaxation of this microstate. At $0 \mathrm{mV}$, four different microscopic states are populated before photooxidation, namely (11111), (11011), (11110), and (11010). The relaxation kinetics at this solution redox potential is thus a superposition of the relaxation kinetics of the states (01111), (01011), (01110), and (01010) in which the electron is ejected from SP. At $150 \mathrm{mV}$, one microstate, namely (11010), is clearly dominating the Boltzmann distribution before photo-oxidation and thus the kinetics at this solution redox potential can again be assigned to one single reaction channel.

Generally, the kinetics trace of one redox-active site is a combination of the kinetic trances of several states. Fig. 9 shows which states contribute to the re-reductionkinetics of SP and at which solution redox potential they do it. The sum of 
with interaction

a) System with four electrons

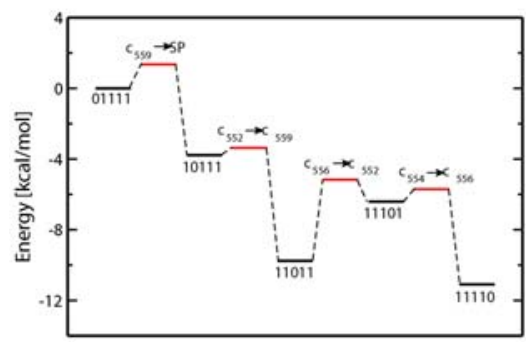

b) System with three electrons

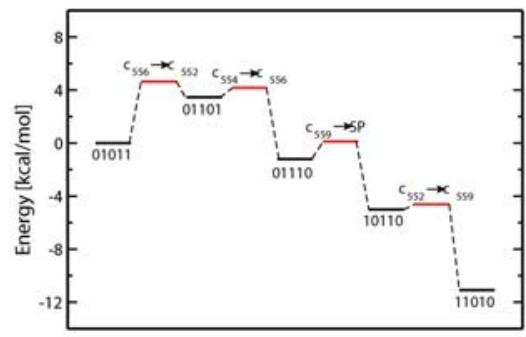

c) System with two electrons

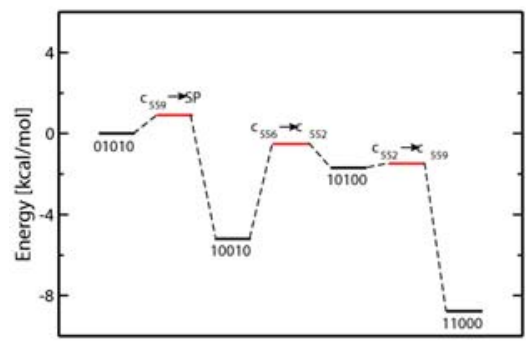

d) System with one electron

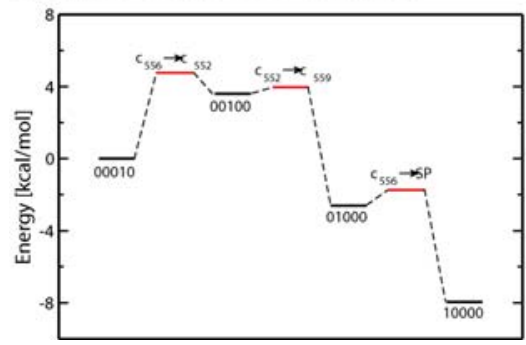

without interaction
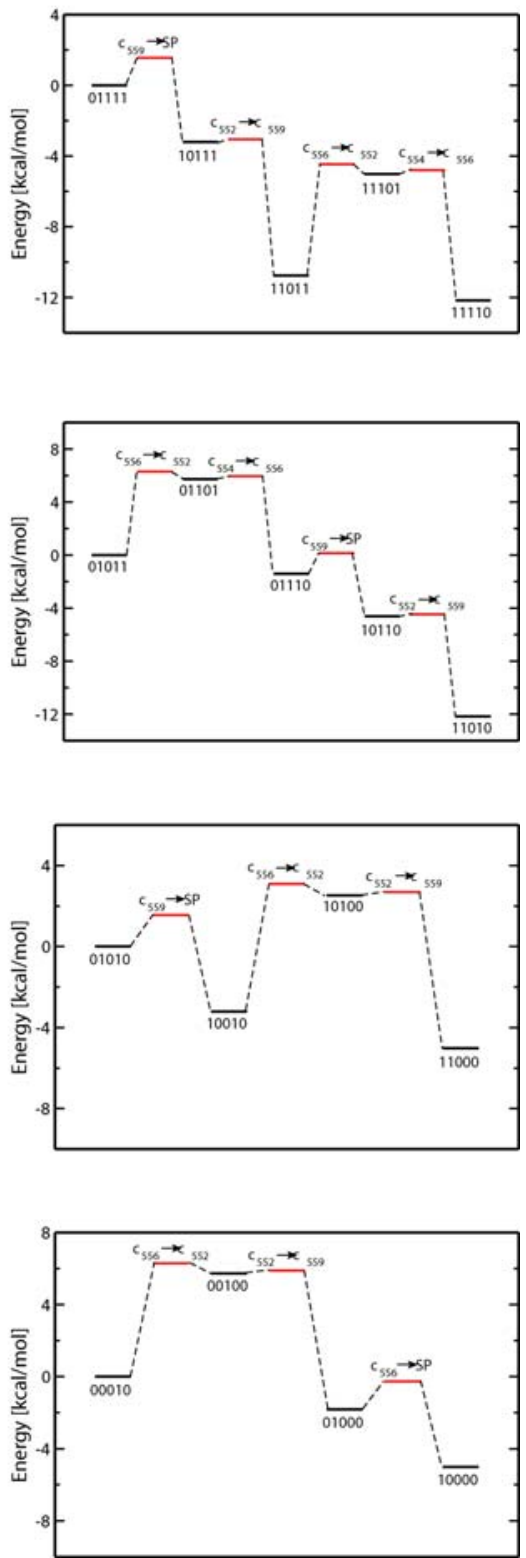

Fig. 7 Energy landscapes of the relaxation kinetics after photo-oxidation of the SP. The left column shows landscapes in which the interaction between the sites is included, the right column shows landscapes in which this interaction is neglected. The landscapes are shown for systems with 4 (a), 3 (b), 2 (c), or 1 (d) electrons. The activation energies (red bars) are calculated from Marcus theory (eqn (17)). The energies of the microstates (black bars) are calculated from eqn (1). The intial state of the reaction sequence was set to zero. At the transition states, it is indicated between which cofactors the electron transfer occurs in the associated reaction step. 


\section{a) State Probability}

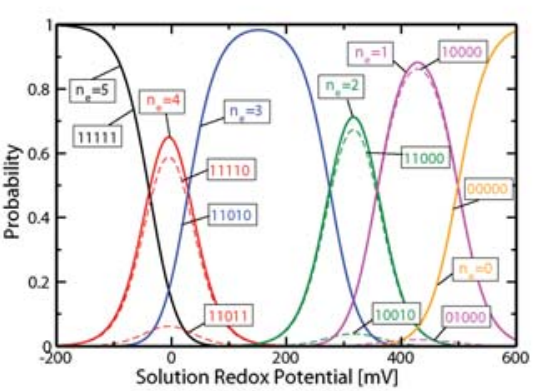

c) Heme $\mathrm{C}_{559}$

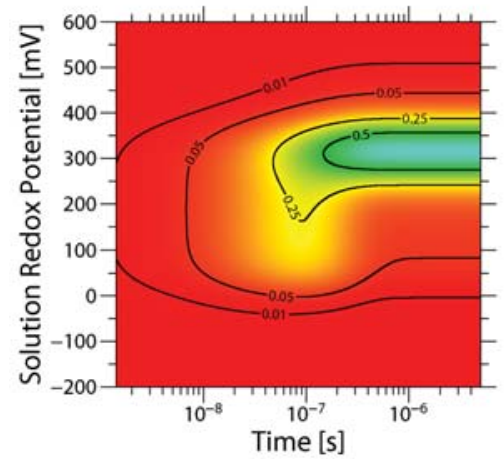

e) Heme $C_{556}$

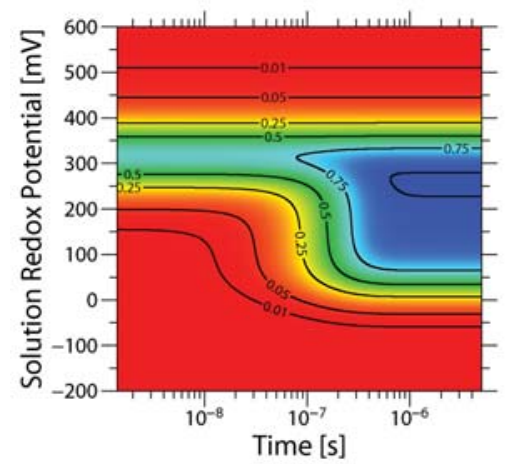

b) Special Pair

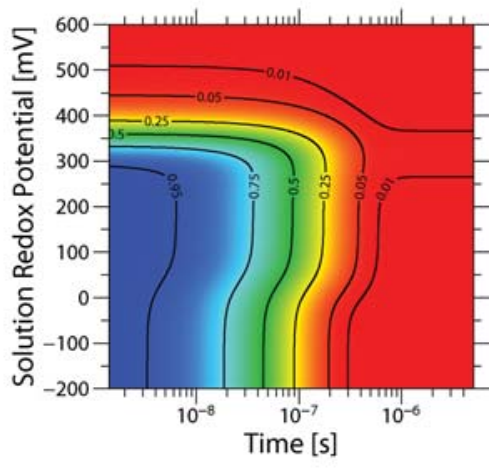

d) Heme $\mathrm{C}_{552}$

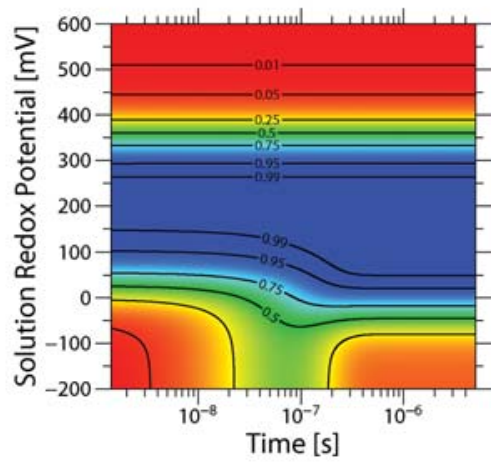

f) Heme $C_{554}$

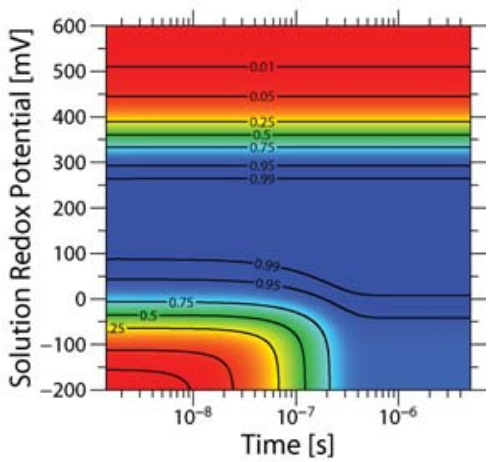

Fig. 8 Time-dependent oxidation probability of the redox-active sites of the RC after photo-oxidation of SP. Initial distributions of the microstates were taken from an equilibrium distribution prior to photo-oxidation of the SP which is shown in (a). The probability of the macroscopic states are shown by solid lines, the probability of the microsciopic states by dashed lines. The oxidation probability of the redox-active sites are shown in (b)-(f). Electron uptake during the equilibration was not considered. The oxidation probabilities of the sites are shown in color (fully oxidized — blue; fully reduced - red) and by contours in the plots. 


\section{a) Special Pair}

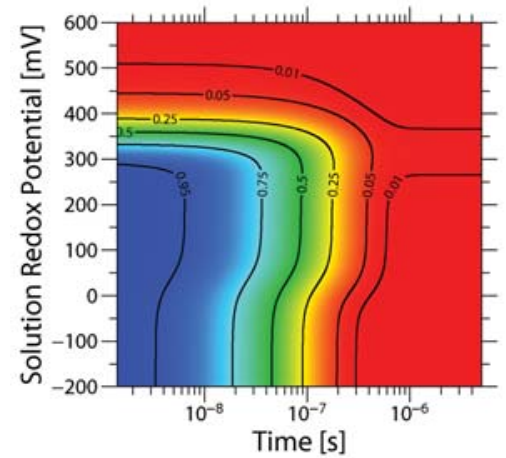

\section{c) State 01110}

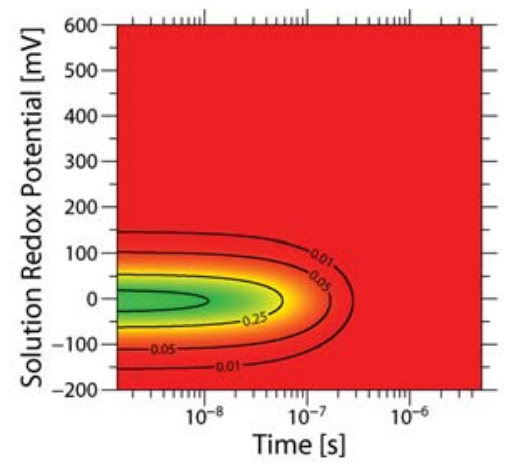

e) State 01010

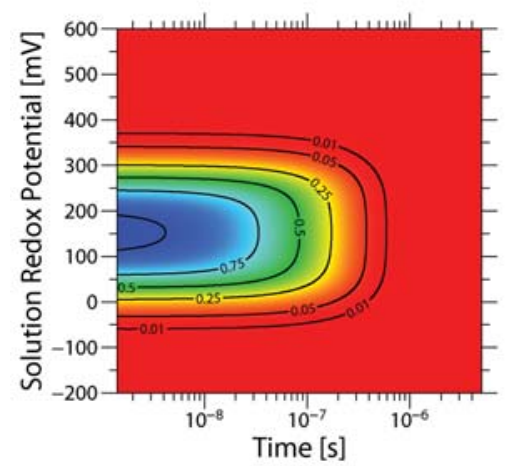

\section{b) State 01111}

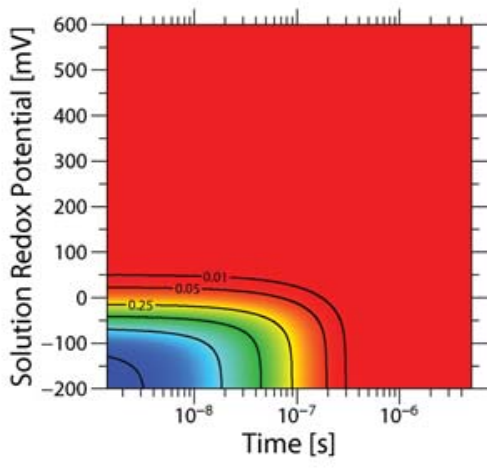

d) State 01011

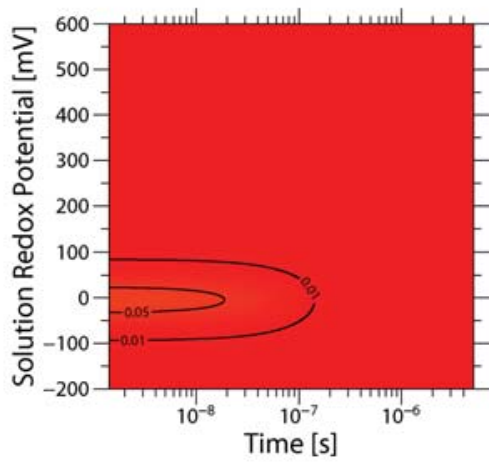

f) State 01000

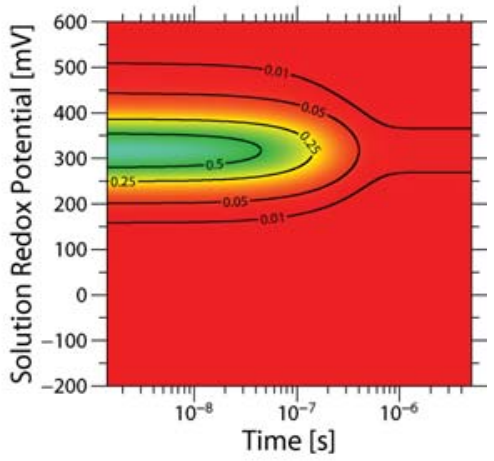

Fig. 9 Re-reduction kinetics of SP in dependence of the redox potential of the solution. The overall re-reduction kinetics of SP shown in (a) is a sum of the kinetic plots of the microstates in (b)-(f). The color and the contours indicate the oxidation probability of SP (blue-fully oxidized; red-fully reduced) or the probabilities of the microstates (blue-populated to $100 \%$; red-unpopulated).

Fig. 9b and c leads to the kinetic trace of SP in Fig. 9a. It can be seen clearly that kinetics at solution redox potentials below $-100 \mathrm{mV}$ is slower than the kinetics at solution redox potentials of about $150 \mathrm{mV}$. The transition between these two kinetics 
occurs at about $0 \mathrm{mV}$. This behavior is due to the mutual interaction between the hemes. As can be seen, many microstates can contribute to the re-reduction kinetics of SP. A deconvolution into individual electron transfer steps is difficult in experiments. Therefore the calculations discussed in this paper provide valuable information for the understanding of complex charge transfer processes.

\section{Conclusions}

In this paper, we show that the interactions between redox cofactors are important for understanding long range electron transfer in proteins involving multiple redox centers. With this work, we complement a previous study ${ }^{16}$ by analyzing the energetics of the electron transfer processes in more detail. The microstate model discussed in this paper is able to provide an appropriate description of the equilibrium and kinetic properties of the relaxation kinetics after photo-oxidation of the special pair in the photosynthetic reaction center. From the energetics of the electron transfer system discussed in this paper, it becomes obvious that it is not correct to assign a fixed redox potential to the redox-active sites in a protein with strongly interacting redox sites. Instead it is possible to describe the energetics of the system by microscopic redox potentials. In this approach the interaction between the redox-active sites is taken into account. The effective redox potentials $\left(\varepsilon_{\mathrm{N}, i}^{\circ}\right.$ and $\left.\varepsilon_{\mathrm{TR}, i}^{\circ}\right)$ can be assigned to the sites of the system.

The approach discussed here is based on an analytical solution of the master equation. However, for large systems, such an analytical solution might not be practical. In such cases, dynamical Monte Carlo simulations can be used to solve the master equation numerically. ${ }^{18}$

The described method offers an approach to describe the thermodynamics and kinetics of complex systems starting from molecular structures. Our approach is thus a first step towards structural systems biology.

\section{Acknowledgements}

This work was supported by the DFG grant UL 174/7-1.

\section{References}

1 E. Bombarda and G. M. Ullmann, J. Phys. Chem. B, 2010, 114, 1994-2003.

2 J. Deisenhofer, O. Epp, K. Miki, R. Huber and H. Michel, J. Mol. Biol., 1984, 180, 385-398.

3 J. Deisenhofer, O. Epp, K. Miki, R. Huber and H. Michel, Nature, 1985, 318, 618-624.

4 J. Deisenhofer, O. Epp, I. Sinning and H. Michel, J. Mol. Biol., 1995, 246, 429-457.

5 S. M. Dracheva, L. A. Drachev, A. A. Konstantinov, A. Y. Semenov, V. P. Skulachev, A. M. Arutjunjan, V. A. Shuvalov and S. N. Zaberezhnaya, Eur. J. Biochem., 1988, 171, 253-264.

6 G. Fritzsch, S. Buchanan and H. Michel, Biochim. Biophys. Acta, Bioenerg., 1989, 977, 157162.

7 J. Ortega and P. Mathis, Biochemistry, 1993, 32, 1141-1151.

8 J. Alric, A. Cuni, H. Maki, K. V. Nagashima, A. Verméglio and F. Rappaport, J. Biol. Chem., 2004, 279, 47849-47855.

9 J. Alric, J. Lavergne, F. Rappaport, A. Verméglio, K. Matsuura, K. Shimada and K. V. Nagashima, J. Am. Chem. Soc., 2006, 128, 4136-4145.

10 Y. Y. Sham, I. Muegge and A. Warshel, Proteins: Struct., Funct., Genet., 1999, 36, 484-500.

11 D. Noy, C. C. Moser and P. L. Dutton, Biochim. Biophys. Acta, Bioenerg., 2006, 1757, 90105.

12 D. Bashford and K. Gerwert, J. Mol. Biol., 1992, 224, 473-486.

13 G. M. Ullmann and E.-W. Knapp, Eur. Biophys. J., 1999, 28, 533-551.

14 G. M. Ullmann, J. Phys. Chem. B, 2000, 104, 6293-6301.

15 A. Ferreira and D. Bashford, J. Am. Chem. Soc., 2006, 128, 16778-90.

16 T. Becker, R. T. Ullmann and G. M. Ullmann, J. Phys. Chem. B, 2007, 111, 2957-2968.

17 M. H. M. Olsson and A. Warshel, Proc. Natl. Acad. Sci. U. S. A., 2006, 103, 6500-6505. 
18 Mirco S. Till, T. E. Torsten Becker and G. M. Ullmann, J. Phys. Chem. B, 2008, 112, 13401-13410.

19 G. M. Ullmann, J. Phys. Chem. B, 2003, 107, 1263-1271.

20 I. M. Klotz Ligand-Receptor Energetics. Wiley \& Sons Inc., New York, 1997.

21 C. Tanford and R. Roxby, Biochemistry, 1972, 11, 2192-2198.

22 C. C. Moser, J. M. Keske, K. Warncke, R. S. Farid and P. L. Dutton, Nature, 1992, 355, 796-802.

23 C. C. Page, C. C. Moser, X. Chen and P. L. Dutton, Nature, 1999, 402, 47-52.

24 R. Marcus, J. Chem. Phys., 1963, 38, 1858-1862.

25 K. E. Sharp, Biophys. J., 1998, 74, 1241-1250.

26 R. A. Marcus and N. Sutin, Biochim. Biophys. Acta, 1985, 811, 265-322.

27 R. J. P. Williams, J. Solid State Chem., 1999, 145, 488-495.

28 M. H. M. Olsson, U. Ryde and B. O. Roos, Prot. Sci., 1998, 7, 2659-2668.

29 U. Ryde and M. H. M. Olsson, Int. J. Quantum Chem., 2001, 81, 335-347.

30 Y. Higuchi, M. Kusunoki, N. Matsuura, N. Yasuoka and M. Kakudo, J. Mol. Biol., 1984, 172, 109-139.

31 A. T. Brünger and M. Karplus, Proteins: Struct., Funct., Genet., 1988, 4, 148-156.

32 B. R. Brooks, R. E. Bruccoleri, B. D. Olafson, D. J. States, S. Swaminathan and M. Karplus, J. Comput. Chem., 1983, 4, 187-217.

33 A. D. MacKerell, D. Bashford, M. Bellott, R. L. Dunbrack, Jr., J. D. Evanseck, M. J. Field, S. Fischer, J. Gao, H. Guo, S. Ha, D. Joseph-McCarthy, L. Kuchnir, K. Kuczera, F. T. K. Lau, C. Mattos, S. Michnick, T. Ngo, D. T. Nguyen, B. Prodhom, W. E. Reiher, B. Roux, M. Schlenkrich, J. C. Smith, R. Stote, J. Straub, M. Watanabe, J. Wiorkiewicz-Kuczera, D. Yin and M. Karplus, J. Phys. Chem. B, 1998, 102, 3586-3616.

34 S. H. Vosko, L. Wilk and M. Nusair, Can. J. Phys., 1980, 1200, 58.

35 P. Perdew, J. A. Chevary, S. H. Vosko, K. A. Jackson, M. R. Pederson, D. J. Singh and C. Fiolhais, Phys. Rev. B: Condens. Matter, 1992, 46, 6671.

36 C. F. Guerra, J. G. Snijders, G. te Velde and E. J. Baerends, Theor. Chem. Acc., 1998, 99, 391-403.

37 C. N. Breneman and K. B. Wiberg, J. Comput. Chem., 1990, 11(3), 361-373.

38 J. L. Chen, L. Noodleman, D. Case and D. Bashford, J. Phys. Chem., 1994, 98, 1105911068.

39 B. Rabenstein, G. M. Ullmann and E. W. Knapp, Biochemistry, 1998, 37, 2488-2495.

40 B. Rabenstein, G. M. Ullmann and E. W. Knapp, Biochemistry, 2000, 39, 10487-10496.

41 A. Taly, P. Sebban, J. C. Smith and G. M. Ullmann, Biophys. J., 2003, 84, 2090-2098.

42 D. Bashford, D. A. Case, C. Dalvit, L. Tennant and P. E. Wright, Biochemistry, 1993, 32, 8045-8056.

43 W. Press, S. Teukolsky, W. Vetterling and B. Flannery, Numerical Recipes in C, Cambridge University Press, 2nd edn, 1992. 